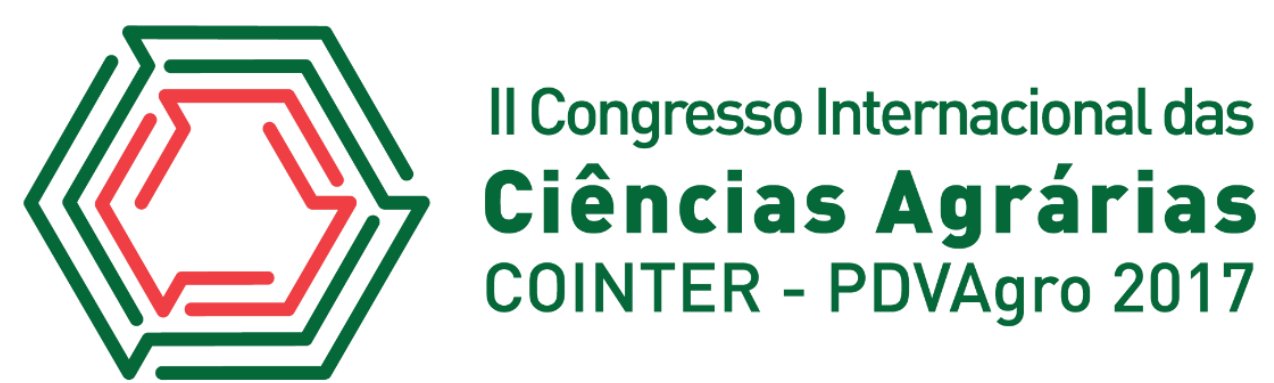

\title{
RELAÇÃO ENTRE GÊNERO E CAUSAS LIMITANTES DE CONSUMO DOS ALIMENTOS ORGÂNICOS NO BRASIL
}

\author{
Apresentação: Pôster \\ Adalgisa Paula de Oliveira Martins ${ }^{1}$; Maria de Fátima Bezerra ${ }^{2}$; Sérgio Marques Júnior ${ }^{3}$; \\ Adriano Henrique do Nascimento Rangel ${ }^{3}$
}

\section{Introdução}

Nos últimos anos, com o aumento da expectativa de vida e o crescimento da cobertura da mídia acerca de questões relacionadas a saúde, ocorreu um estímulo nos diversos setores de produção de alimentos, ampliando assim a oferta de produtos que proporcionem benefícios fisiológicos além de apresentarem importância nutricional e propriedades sensoriais agradáveis, melhorando a saúde dos consumidores. O sistema de produção orgânica, além de proporcionar benefícios ao meio ambiente, se preocupa com a promoção de relações equitativas e boa qualidade de vida para todos os envolvidos, considerando os impactos ambientais e sociais. Contudo, existem barreiras que implicam na baixa demanda no mercado, quando comparado com o convencional, em função do preço elevado, consumo insuficiente, pouca disponibilidade, curto prazo de validade e baixa exposição de produtos nos estabelecimentos comerciais. Diante disso, o presente trabalho teve o intuito de investigar o comportamento de gêneros (feminino e masculino) em relação a causas limitantes de consumo de alimentos orgânicos.

\section{Fundamentação Teórica}

A avaliação das necessidades do consumidor e de sua conduta na ocasião da compra são imprescindíveis no fornecimento de dados para o desenvolvimento de novos produtos e também para manutenção das estratégias de marketing de forma a alcançar a satisfação do consumidor e, consequentemente, consolidar o produto no mercado (PINHEIRO et al., 2011). Minim (2013) destaca

\footnotetext{
${ }^{1}$ Bolsista de Mestrado/Capes no Programa de Pós-Graduação em Produção Animal, UFRN, martins.adalgisa@yahoo.com.br

2 Bolsista PNPD/Capes no Programa de Pós-Graduação em Produção Animal, UFRN, fatimabezerra1979@gmail.com

${ }^{3}$ Professor da UFRN, sergio@ct.ufrn.br

${ }^{3}$ Professor da UFRN, adrianohrangel@yahoo.com.br
} 
que a satisfação do consumidor está baseada nos parâmetros de qualidade do produto, os quais pode variar de pessoa para pessoa em função de fatores como: características sensoriais do alimento, método de preparo, custo, sazonalidade, além das peculiaridades do indivíduo aliadas à idade, sexo, educação, renda, grau de urbanização em que o sujeito está inserido, ambiente de trabalho e da estação do ano.

O terceiro milênio tem sido marcado por um aumento na expectativa de vida e uma grande cobertura da mídia acerca de questões relacionadas a saúde, estimulando os diversos setores de produção de alimentos a ampliar a oferta de produtos que além de apresentar importância nutricional e propriedades sensoriais agradáveis, proporcionem benefícios fisiológicos melhorando a saúde dos consumidores (SILVA, BARREIRA \& OLIVEIRA, 2016). Nesse contexto, o conceito de agricultura orgânica tem ganhado espaço no mercado por se tratar de um sistema de produção que preserva a saúde dos solos, dos ecossistemas e das pessoas, tendo como objetivo produzir alimentos saudáveis e de qualidade, sem a utilização de produtos químicos sintéticos (FAO, 2016; IFOAM, 2016). O sistema de produção orgânica, além dos benefícios proporcionados ao meio ambiente, se preocupa com a promoção de relações equitativas e boa qualidade de vida para todos os envolvidos (IFOAM, 2016).

Os alimentos orgânicos se destacam pela salubridade, caráter ecológico, sabor agradável, segurança alimentar e garantia de qualidade, entretanto, existem barreiras que implicam na baixa demanda no mercado, quando comparado com o convencional, em função do preço elevado, consumo insuficiente, pouca disponibilidade, curto prazo de validade e baixa exposição de produtos nos estabelecimentos comerciais (BRYLA, 2016). Com relação ao perfil de consumidores de alimentos orgânicos, estudos realizados em alguns países demonstram que a escolha por esses produtos está mais relacionada a valores hedônicos como bem-estar próprio e de sua família do que a valores éticos relacionados aos efeitos sociais e ambientais do sistema de produção orgânico (ANNUNZIATA \& VECCHIO, 2016).

\section{Metodologia}

Durante todo o mês de março de 2017, foi transmitido aos voluntários via redes sociais (WhatsApp ${ }^{\circledR}$ e Facebook®) e via e-mail uma investigação do perfil de consumidor mediante aplicação de questionário abrigado em site na web, o Google Formulários® (docs.google.com/forms/). Foram realizadas duas questões abordando causas de alimentos orgânicos com respostas estruturadas em escalas de avaliação numérica (de 0 a 10). Foi realizada a seguinte pergunta: quais as principais causas que limitam seu consumo de alimentos orgânicos? Os 
respondentes avaliaram as seguintes variáveis: o preço elevado, falta de interesse, qualidade inferior, baixa variedade, falta de confiança, difícil acesso, oferta irregular e poucos estabelecimentos comercializam. Após a finalização do teste, as respostas foram coletadas diretamente no site, transferidas para o Excel® e, posteriormente os dados foram analisados pelo software estatístico IBM SPSS22 Statistics ${ }^{\circledR}$ mediante o Teste-T para amostras independentes.

\section{Resultados e Discussões}

Na Tabela 1 é analisada a relação de gênero (feminino e masculino) com as principais causas que limitam o consumo de alimentos orgânicos como preço elevado, falta de interesse, qualidade inferior, baixa variedade, falta de confiança, dificuldade de acesso, oferta irregular ou o fato de poucos estabelecimentos comercializarem. O nível de concordância foi avaliado em uma escala numérica, onde 0 significava "discordo totalmente" e 10 significava "concordo totalmente".

Tabela 1. Relação entre gênero e causas limitantes de consumo de alimentos orgânicos.

\begin{tabular}{|c|c|c|c|}
\hline VARIÁVEIS & $\begin{array}{c}\text { MASCULINO } \\
(\mathbf{N}=\mathbf{3 8 4})\end{array}$ & $\begin{array}{c}\text { FEMININO } \\
\qquad(\mathrm{N}=\mathbf{8 4 6})\end{array}$ & $p$-valor \\
\hline Preço elevado & $8,01 \pm 2,32$ & $8,24 \pm 2,36$ & 0,109 \\
\hline Falta de interesse & $3,29 \pm 3,20$ & $2,34 \pm 3,09$ & 0,000 \\
\hline Qualidade inferior & $1,59 \pm 2,53$ & $0,92 \pm 1,99$ & 0,000 \\
\hline Baixa variedade & $4,05 \pm 3,23$ & $3,65 \pm 3,29$ & 0,046 \\
\hline Falta de confiança & $3,57 \pm 3,36$ & $3,33 \pm 3,33$ & 0,262 \\
\hline Difícil acesso & $6,89 \pm 2,90$ & $6,92 \pm 2,94$ & 0,845 \\
\hline Oferta irregular & $6,42 \pm 2,90$ & $6,18 \pm 3,08$ & 0,180 \\
\hline Poucos estabelecimentos comercializam & $7,53 \pm 2,55$ & $7,82 \pm 2,51$ & 0,057 \\
\hline
\end{tabular}

Resultados apresentados como média \pm desvio padrão.

Na referida Tabela, observa-se a diferença estatística na baixa variedade entre as principais causas que limitam os respondentes no consumo dos alimentos orgânicos, os resultados mostram que homens concordam em maior número com esta afirmativa em relação às mulheres [M (homens): 4,05; $\mathrm{M}$ (mulheres): 3,65]. Comportamento semelhante foi observado para a falta de interesse em consumir alimentos orgânicos, onde homens apresentaram um grau de concordância significativamente maior que as mulheres [M (homens): 3,29; $\mathrm{M}$ (mulheres): 2,34], tal resultado corrobora com estudos conduzidos por Hempel e Hamm (2016), os quais apontam que os 
consumidores do sexo feminino são frequentemente identificados como tendo maior preferência para os alimentos orgânicos quando comparados aos homens. Também houve diferença no grau de concordância para qualidade inferior dos alimentos orgânicos, onde os homens se sobressaíram em relação às mulheres [M (homens: 1,59; $\mathrm{M}$ (mulheres): 0,92]. As médias apresentaram escalas mais altas (de maior grau de concordância) nas perguntas sobre falta de interesse e poucos estabelecimentos comercializam, onde o preço elevado se destaca como uma das principais causas limitantes no consumo de alimentos orgânicos, não havendo diferença significativa entre homens e mulheres [M (homens): 8,01; M (mulheres): 8,24] e afirmaram concordar totalmente com o fato de que se poucos estabelecimentos comercializarem os produtos orgânicos limita o consumo destes produtos, não havendo diferença significativa entre homens e mulheres [M (homens): 7,53; $\mathrm{M}$ (mulheres) 7,82]. Observou-se que as médias apresentaram escalas mais baixas (maior discordância) nas afirmações sobre falta de interesse, qualidade inferior e falta de confiança, onde o maior número de respondentes afirmou discordar totalmente com as afirmações citadas. Pinheiro (2011) ressaltou que os aspectos apontados como de maior importância para os consumidores no momento da compra foram parâmetros nutricionais e preço.

\section{Conclusões}

As principais causas indicadas como fatores limitantes do consumo de orgânicos entre homens e mulheres foram o preço elevado e a presença de poucos estabelecimentos de comercialização. A falta de interesse e a baixa variedade constituíram-se em fatores de maior limitação entre os homens quando comparado com as mulheres, enquanto para estas, a qualidade inferior do produto tem importância mais negativa.

\section{Referências}

ANNUNZIATA, A.; VECCHIO, R. Organic farming and sustainability in food choices: an analysis of consumer preference in Southern Italy. Agriculture and Agricultural Science Procedia, v.8, p.193-200, 2016

BERNACCHIA, R.; PRETI, R.; VINCI, G. Organic and conventional foods: Differences in nutrientes. Italy J. Food Sci., v. 28. p.565- 578, 2016.

BRYLA, P. Organic food consumption in Poland: Motives and barriers. Appetite. v.105, p. 737-746, 2016. 
FAO. Food and agriculture Organization of the Unites Nation. What is organic agriculture? Disponível em http://www.fao.org/organicag/oa-faq/oa-faq1/en. Consulta realizada em 21 de Setembro de 2016.

HEMPEL, C.; HAMM, U. How important is local to organic-minded consumers? Appetite, v.96, n.309-318, 2016.

IFOAM. Organic International. Organic Basic. Disponível em: https://www.ifoam.bio/en/ourlibrary/organic-basics. Consulta realizada em 21 de Setembro de 2016.

MINIM, V.P.R. Análise sensorial: estudo com consumidor. 3 ed. Viçosa: UFV, 2013.

Pinheiro, F. A., CARdoso, W. S., ChAVES, K. F., OliveirA, A. S. B., RiOS, S. A. Consumers profile in relation to food quality and purchase habits. UNOPAR, Científica Ciências Biológicas e da Saúde. 2011. v.13, n.2, p.95-102.

SILVA, B. V., BARREIRA, J. C. M., OLIVEIRA, M. B. P. P. Natural phytochemicals and probiotics as bioactive ingredientes for functional foods: Extraction, biochemistry and protected-delivery Technologies. Trends in Food Science \& Technology. 2016. V.50, p.144-158. 